\title{
Domain-wall scattering in epitaxial FePd ordered alloy films with perpendicular magnetic anisotropy
}

\author{
D. Ravelosona, * A. Cebollada, and F. Briones \\ Instituto de Microelectronica de Madrid, Isaac Newton 8, PTM, 28760 Tres Cantos (Madrid), Spain \\ C. Diaz-Paniagua, ${ }^{\dagger}$ M. A. Hidalgo, and F. Batallan \\ Instituto de Ciencas de Materiales de Madrid, CSIC, Cantoblanco, 28049 Madrid, Spain
}

(Received 26 October 1998)

\begin{abstract}
Domain-wall scattering in a FePd-ordered alloy film with strong perpendicular magnetic anisotropy has been investigated using magnetoresistance measurements on a whole hysteresis loop and as a function of temperature. A strong correlation between the hysteretic behavior of the resistivity due to domain walls and the magnetization process is found. The data are well described by a spin-dependent electron scattering model. This study establishes that this phenomenon can be used to identify directly magnetization reversal dynamics and electronic transport characteristics in materials with high magnetic anisotropy. [S0163-1829(99)07805-4]
\end{abstract}

Domain-wall (DW) scattering as well as the extraordinary Hall effect (EHE) are physical properties that reflect both the electronic transport characteristics and the magnetic nature of a ferromagnetic material. The study of these phenomena is very powerful because it can provide direct information about magnetization reversal or domain-wall dynamics. Whereas the EHE has been extensively investigated in ferromagnetic metals and alloys, ${ }^{1}$ and recently in magnetic multilayers, ${ }^{2}$ scattering by DW has not received proper attention (except the early work of Cabrera and Falicov ${ }^{3}$ and Berger $^{4}$ ) due to the difficulty to measure directly and unambiguously the contribution of such small magnetic objects $(5-50 \mathrm{~nm})$.

Recently the additional resistivity due to DW scattering was calculated by Levy et al. ${ }^{5}$ with the same Hamiltonian as used in the giant magnetoresistance (GMR) two-spinchannel model. They predicted that the resistivity is enhanced in the presence of domain walls. Such a prediction has been experimentally reported by Gregg et al. ${ }^{6}$ who obtained a direct indication of the DW contribution to the resistivity in a striped domain structure in a Co film with large perpendicular anisotropy. The interplay between magnetic and transport properties was not clear because the hysteresis which is an indication of the magnetic nature of the phenomenon, was not investigated in detail. In addition, no temperature dependence of the phenomenon was studied. Also, another recent experiment ${ }^{7}$ using $\mathrm{MR}$ measurements in $\mathrm{Fe}$ nanowires reports that the resistivity decreases in the presence of DW's. While a negative contribution is consistent with quantum transport theory, ${ }^{8}$ the results are difficult to reconcile with this theory. In Ref. 7 the DW contribution has not been directly determined but isolated from that due to anisotropic magnetoresistance (AMR) and ordinary MR (Lorentz).

This paper is devoted to the detailed investigation of DW scattering in a thin ferromagnetic film. We use a striped domain structure in a FePd ordered alloy film with strong perpendicular magnetic anisotropy. We observe directly the extra resistivity due to DW on a complete field hysteresis loop and for a wide range of temperatures. We find that the resis- tivity is enhanced in the presence of domain walls. We compare our results to a recent model for wall scattering.

The samples were prepared on polished $\mathrm{MgO}(100)$ substrates by a UHV triode-sputtering system. They consist of a $1000 \AA$ thick epitaxial $\mathrm{Fe}_{50} \mathrm{Pd}_{50}(001)$ layer grown on a 350 $\AA$ thick Pt (001) buffer layer and capping by $50 \AA$ of Pt. In a previous study, we demonstrated that $\mathrm{Fe}_{50} \mathrm{Pd}_{50}$ films with a high degree of chemical order can be obtained by tuning the growth temperature. 9 Figure 1 shows the magnetization curves measured with the field applied either parallel or perpendicular to the film plane for an ordered alloy film. The values of the demagnetizing energy $2 \pi M_{s}^{2}$, the anisotropy uniaxial constant $K_{U}$, and the estimated wall thickness $\delta_{w}$ are listed in Table I for the FePd-ordered film and for a typical Co film. ${ }^{10}$ The FePd $L_{10}$ ordered tetragonal structure leads to a magnetocrystalline anisotropy $K_{u}$ of $1 \times 10^{7}$ $\mathrm{erg} / \mathrm{cm}^{3}$ larger than the demagnetizing energy, which keeps the magnetization perpendicular to the sample. This large anisotropy gives rise to a small wall thickness of $100 \AA$. For comparison the wall thickness in Co films of Ref. 10 is estimated to be $150 \AA$. In the inset to Fig. 1, it is shown a detail of the positive branch of the magnetization of the ordered alloy. The shape of the curve indicates that the film is not homogeneously magnetized. From Kerr microscopy, the magnetic structure is seen to be divided into alternatively small up and down magnetized domains (stripe domains) with a characteristic width of $120 \mathrm{~nm}$. These values are in agreement with those measured recently in FePd films grown by molecular-beam epitaxy. ${ }^{11}$

The magnetoresistance was measured in a high-field cryostat by the typical four-point technique with the current applied in the plane of the films and the magnetic field perpendicular to the samples. Figure 2(a) reports the typical resistivity loop for the sample of Fig. 1 measured at $4.2 \mathrm{~K}$. Generally, as the current and the magnetization remain perpendicular on the whole field loop the anisotropic magnetoresistance (AMR) is absent. However, even if the anisotropy is large, closure structure at the surface with the magnetization in the plane of the sample can give an AMR 


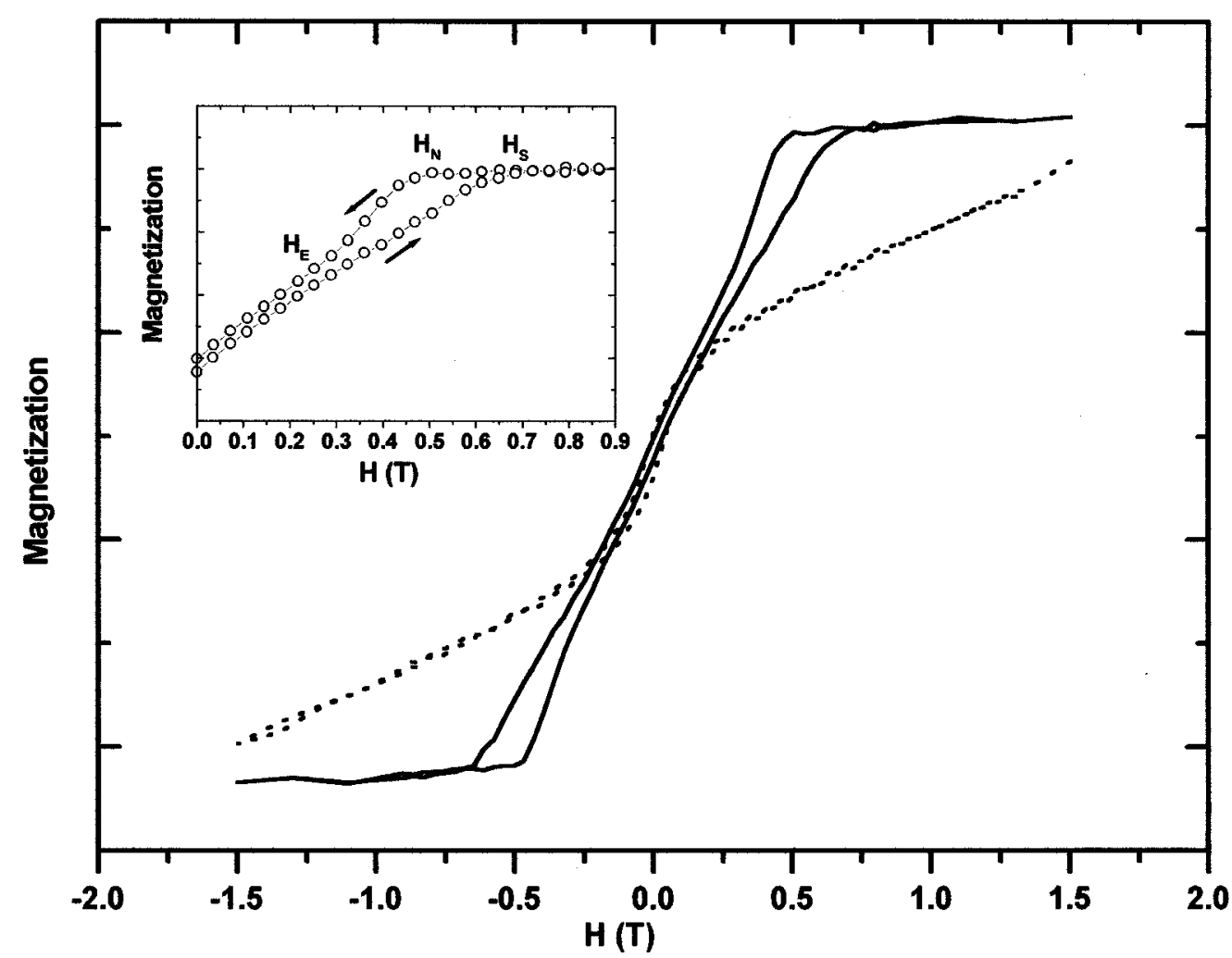

FIG. 1. $M-H$ curves determined by VSM measurement with the field perpendicular (solid line) and parallel (dot line) to the film plane for a FePd-ordered alloy film with $S=0.8$. The order parameter $S$ has been determined by x-ray measurements $(S=0$ for a disordered alloy and $S=1$ for a perfectly ordered alloy) (Ref. 9). The inset shows the positive field branch of the perpendicular magnetization of the ordered alloy where $H_{N}$ is the nucleation field, $H_{S}$ is the field of saturation, and $H_{E}$ is the field of equilibrium.

contribution to the MR. ${ }^{7}$ By measuring the MR in transverse and longitudinal geometries, ${ }^{12}$ we have verified that such an AMR contribution was negligible in our sample. In addition we found that ordinary MR (Lorentz) only contributes to the resistivity above 2 T. The extra resistivity seen in Fig. 2(a) only arise from scattering at the domain walls. As seen in Fig. 2(b) which presents a detail of the positive branch of Fig. 2(a), the most striking feature is the hysteretic behavior of the resistivity very similar to the magnetization one. The characteristic fields of the hysteresis, $H_{E}, H_{S}$, and $H_{N}$ as indicated in the inset to Fig. 1 and in Fig. 2(b) are identical for both processes. Interestingly, the resistivity exhibits a singularity at $H_{N}$ as well as the magnetization loop. Here let us discuss the interplay between magnetic and transport properties. The zero-field state is a maze configuration of

TABLE I. Values of the uniaxial anisotropy constant $K_{U}$, the demagnetizing energy $2 \pi M_{S}^{2}$ and the wall thickness $\delta_{w}$ for a FePdordered alloy and for a typical Co film (Ref. 10). The wall thickness $\delta_{w}$ is calculated as $\delta_{w}=\pi\left(J / K_{U}\right)^{1 / 2}$ using $J=10^{-6} \mathrm{erg} / \mathrm{cm}$ for the exchange coupling constant. For the FePd film, the magnetization was determined by vibrating sample magnetometer (VSM) and the uniaxial anisotropy constant by Torque measurement at $300 \mathrm{~K}$.

\begin{tabular}{lccc}
\hline \hline & $K_{U}\left(\mathrm{erg} / \mathrm{cm}^{3}\right)$ & $2 \pi M_{S}^{2}\left(\mathrm{erg} / \mathrm{cm}^{3}\right)$ & $\delta_{w}(\AA)$ \\
\hline FePd & $1 \times 10^{7}$ & $9 \times 10^{6}$ & 100 \\
Co & $4.6 \times 10^{6}$ & $1.3 \times 10^{7}$ & 150 \\
\hline \hline
\end{tabular}

perpendicular strip domain (stage 1). The magnetoresistance plateau seen in Fig. 2 which corresponds to the linear increase of $M$ with $H$ as indicated in Fig. 1 is characteristic of easy domain-wall propagation: the width $d_{1}$ of the domains with their magnetization vector parallel to the applied field increases and the width $d_{2}$ of the reversed domain decreases. The fact that the resistivity remains constant indicates that the density of the domain walls is conserved, i.e., $d_{1}+d_{2}$ remains constant in such process (stage 2). By further increasing the field, a strong decrease of the resistivity is observed because near saturation the walls are destroyed due to a rapid enhancement of $d_{1}$ (stage 3 ). This behavior is consistent with the model developed by Kooy and Enz ${ }^{13}$ to interpret the process of domain motion for films with perpendicular anisotropy. Now starting from saturation and decreasing the field (stage 4), a steep enhancement of the resistivity is observed at the nucleation field $H_{N}$. This singularity of the resistivity shows that a great number of domain walls are suddenly created (stage 5). It is interesting to note that this drop corresponds to the $30 \%$ of the total magnetoresistance observed, which indicates that the domain walls are created over a large surface of the sample. Cape et $a l .{ }^{14}$ (see also Hehn et al. ${ }^{10}$ ) established that the singularity in the magnetization loop for films with an easy-axis perpendicular to the film plane can be ascribed to a sudden nucleation of magnetic bubbles with opposite magnetization. Also seen in Fig. 2, the slight increase of the resistance between $H_{N}$ and $H_{E}$ could indicate the existence of a disper- 

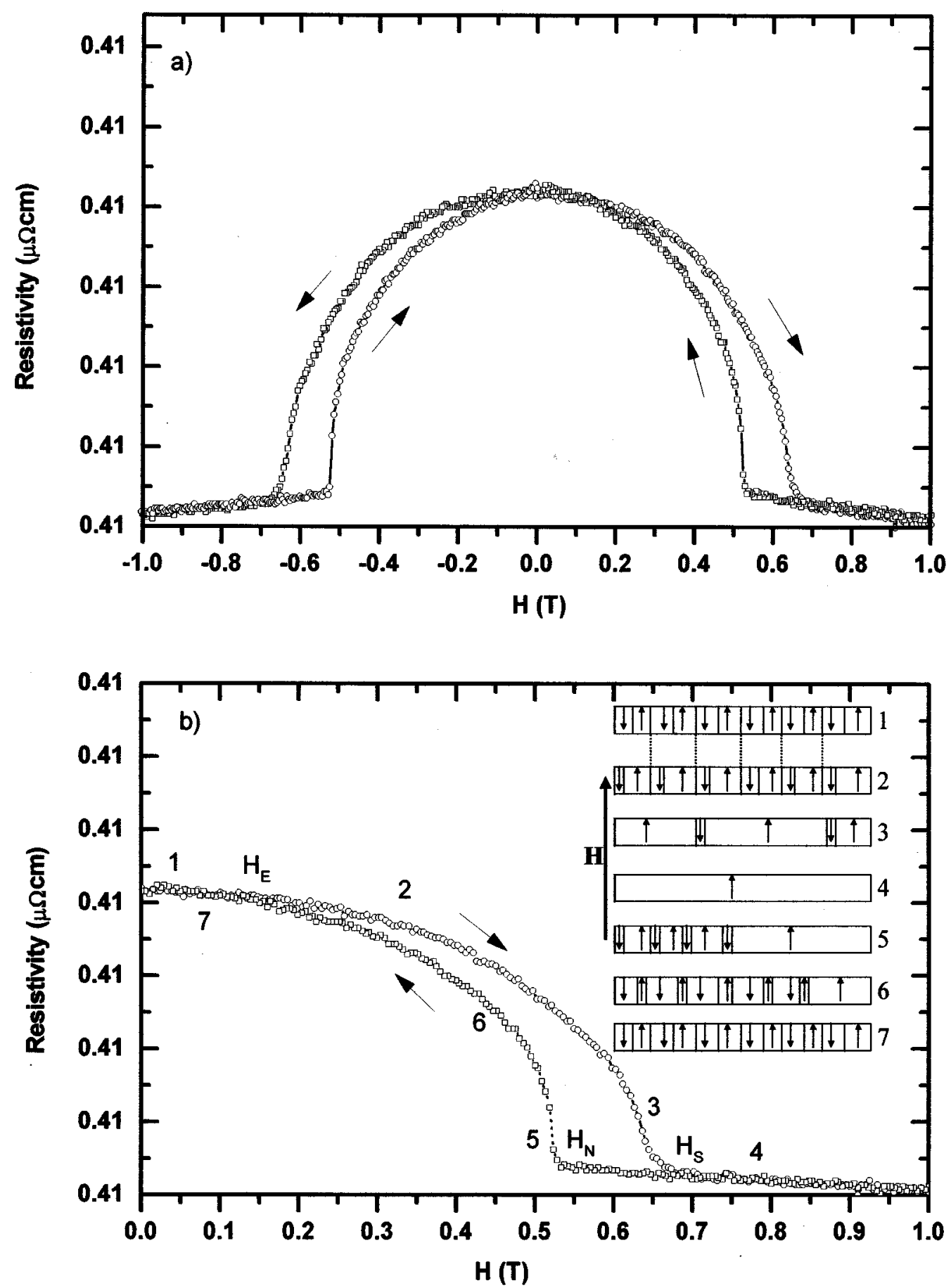

FIG. 2. MR of the ordered film of Fig. 1 at $4.2 \mathrm{~K}$. (a) complete hysteresis loop, (b) detail of the positive branch. The inset to Fig. 2(b) indicates schematically the wall dynamic behavior as described in the text. The slight decrease of the resistivity above $H_{N}$ is probably due to magnon damping (increase in $M$ above $M_{S}$ caused by the applied field). Above $2 \mathrm{~T}$ the ordinary MR (Lorentz) that increases the resistivity is dominant.

sion in nucleation fields of bubbles. ${ }^{15}$ However, the fact that the resistivity loop only shows one singularity could also indicate that only one nucleation event occurs followed by a propagation over the whole sample (stage 6). At $H_{E}=0.2 \mathrm{~T}$, the bubble domain occupies the entire sample and the equilibrium state is reached (stage 7). For comparison, we have included in Fig. 3 the extra resistivity due to DW scattering for a FePd ordered film $(S=0.85)$ with larger crystalline disorder (but with similar chemical order) than the alloy of Fig. 2. This was obtained by growing the film at a higher temperature which results in a grain segregation. Here there is no evidence of steep transition at $H_{N}$ because the magnetization reversal is dominated by a nucleation process at the expense of the easy domain-wall propagation depicted in Fig. 2(a). Consequently a large number of small inverted domains is created between $H_{N}$ and $H_{E}$ with a large distri- 


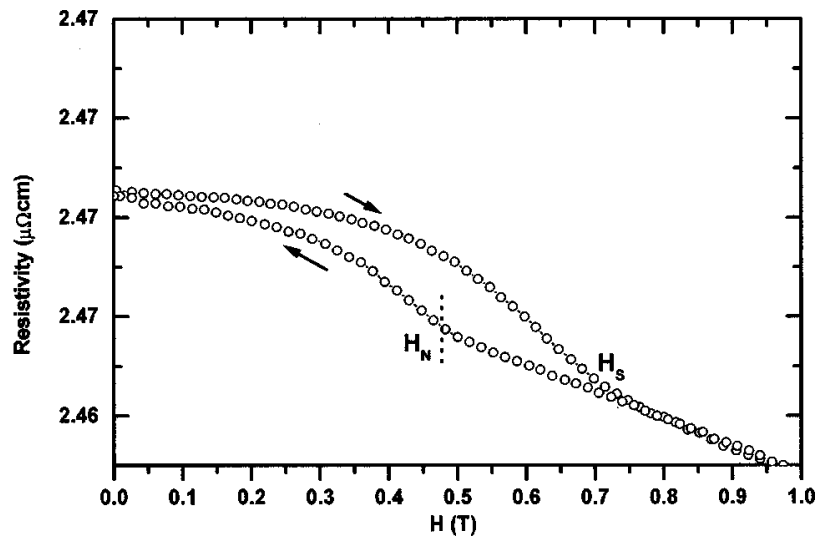

FIG. 3. MR of a FePd-ordered film $(S=0.85)$ with large crystalline disorder at $4.2 \mathrm{~K}$. The chemical order and the perpendicular magnetic anisotropy are similar to the alloy of Fig. 2.

bution of nucleation fields which results in a very slight increase of the resistance. Results of Figs. 2 and 3 establish that wall scattering can be used for detecting reversal magnetization dynamics in materials with high perpendicular anisotropy.

From the measurement of Fig. 2, we find that the extra resistivity ${ }^{5}$ of a DW over that of the pure FePd alloy $\Delta \rho_{\mathrm{FePd}}=\rho_{w}-\rho_{0}$ with $\rho_{w}$ the resistivity of a DW and $\rho_{0}$ the resistivity of the sample without DW is about $1 \mu \Omega \mathrm{cm}$. This gives a MR ratio $R_{w}=\Delta \rho_{\mathrm{FePd}} / \rho_{0}$ of about $6 \%$ (in total the measured MR ratio of the sample $R=\left(\delta_{w} / d\right) R_{w}$ with $d$ the domain size is $\sim 0.5 \%$ ). Note that the diamagnetic and the paramagnetic mechanisms invoked by Cabrera $\mathrm{et} \mathrm{al.}{ }^{3}$ cannot account for the additional resistivity observed. The resistivity due to DW scattering was calculated in the presence of stripe domains by Levy and Zhang ${ }^{5}$ with the same Hamiltonian as used to explain giant magnetoresistance taking account the admixture of spin states due to the twisting of magnetization in domain walls. They found the following analytical expression for the magnetoresistance ratio with the current parallel to the DW (CIW) at low temperatures:

$$
R_{\mathrm{CIW}}=\left(\rho_{\mathrm{CIW}}-\rho_{0}\right) / \rho_{0}=\frac{\xi^{2}}{5} \frac{\left(\rho_{0}^{\uparrow}-\rho_{0}^{\downarrow}\right)^{2}}{\rho_{0}^{\uparrow} \rho_{0}^{\downarrow}},
$$

where $\xi=\pi \hbar^{2} k_{F} / 4 m \delta_{w} J$ and $J$ denotes the exchange splitting, $\rho_{\text {ciw }}$ is the resistivity of a DW and $\rho_{0}^{S}$ the resistivity for spin $s$ of the ferromagnet. For a current perpendicular to the domain wall (CPW), they predicted that the ratio of CPW and CIW magnetoresistance due to DW is given by

$$
R_{\mathrm{CPW}} / R_{\mathrm{CIW}}=3+10 \sqrt{\frac{\rho_{0}^{\uparrow} \rho_{0}^{\downarrow}}{\rho_{0}^{\uparrow}+\rho_{0}^{\downarrow}}} .
$$

With the common parameter for $\mathrm{Co}, \mathrm{Fe}$, and Ni ferromagnets, $k_{F}=1 \AA^{-1}, J=0.5 \mathrm{eV}$, and $\rho_{0}^{\downarrow} / \rho_{0}^{\uparrow}=5-20$ and for a wall $100 \AA$ thick, $R_{\mathrm{CIW}}$ ranges from 0.5 to $2.7 \%$ and $R_{\mathrm{CPW}}$ from 3.5 to $14 \%$. Here, the magnetoresistance ratio $R_{w}$ was measured with a maze configuration of domain, which can be viewed as a mixed of both geometry. In this case, the analytical expression for $R_{w}$ can be approximated by

$$
R_{W}=\left(R_{\mathrm{CIW}}+R_{\mathrm{CPW}}\right) / 2
$$

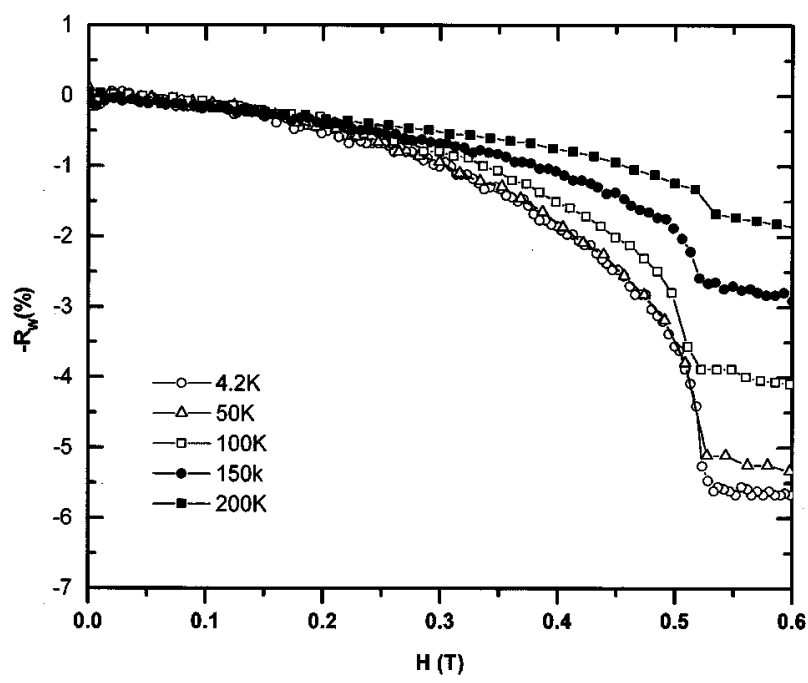

FIG. 4. Magnetoresistance ratio for a DW $-R_{w}$ of the FePdordered alloy film of Fig. 2 measured by decreasing the field from saturation to zero field as a function of temperature. For temperatures higher than $200 \mathrm{~K}$ and above $H_{S}$ magnon damping gives a strong negative contribution to the resistivity. As a consequence although DW scattering is still present at $300 \mathrm{~K}$, it is not possible to discern the transition fields $H_{N}$ and $H_{S}$.

with $R_{\mathrm{CIW}}$ and $R_{\mathrm{CPW}}$ given by Eqs. (1) and (2), respectively, which ranges from $2 \%$ to $8.5 \%$. Our value of $6 \%$ at $4.2 \mathrm{~K}$ is consistent with these predictions. From Eq. (3) we deduce a spin asymmetry of scattering $\rho_{0}^{\downarrow} / \rho_{0}^{\uparrow} \sim 16$. However, it is expected that $\rho_{0}^{\downarrow} / \rho_{0}^{\uparrow}$ ranges from $1 / 11(\mathrm{Mn})$ to 9 (Ir) at low temperatures for dilute impurities in $\mathrm{Fe} .{ }^{16}$ Here, the high scattering ratio found at $4.2 \mathrm{~K}$ could be due to the use in Eq. (1) of the wall thickness value estimated at $300 \mathrm{~K}$. As the uniaxial anisotropy is expected to be stronger at low temperatures, the domain walls are generally thinner at $4.2 \mathrm{~K}$ than at $300 \mathrm{~K}$. Then from Eq. (1), we deduced that we overestimated the spin asymmetry of scattering. Also, the ratio $\rho_{0}^{\downarrow} / \rho_{0}^{\uparrow}$ is expected to be proportional to $n_{\downarrow}\left(E_{F}\right) / n_{\uparrow}\left(E_{F}\right)$, where $n_{\uparrow(\downarrow)}\left(E_{F}\right)$ is the density of states at the Fermi energy for spin up (down). By allowing $\mathrm{Fe}$ or Co with equiatomic composition of $\mathrm{Pd}$ or $\mathrm{Pt}$, the $d$ bands of $\mathrm{Fe}(\mathrm{Co})$ and $\mathrm{Pd}(\mathrm{Pt})$ are strongly hybridized in the majority-spin states because they coincide in energy. ${ }^{17}$ Both majority $d$ bands are completely filled which leads to a small value of $n \uparrow\left(E_{F}\right)$. On the other hand, the hybridization between the $\mathrm{Fe}(\mathrm{Co})$ and $\mathrm{Pd}$ $(\mathrm{Pt})$ minority $d$ bands is weak because of their separation in energy. The tetragonal distortion related to ordering removes a portion of the minority $d$ bands of $\mathrm{Fe}(\mathrm{Co})$ above $E_{F} \cdot{ }^{18}$ This results in an increase of $n_{\downarrow}\left(E_{F}\right)$ and then to a large value of the spin asymmetry for ordered alloys.

The behavior of the magnetoresistance ratio $R_{w}$ by decreasing the field for temperatures ranging from 4.2 to $200 \mathrm{~K}$ is shown in Fig. 4. The characteristic low-field plateau and the singularity of the resistance are present for all the temperatures investigated. The most striking feature here is the strong temperature dependence of $R_{w}$ which ranges from $6 \%$ at $4.2 \mathrm{~K}$ to $2 \%$ at $200 \mathrm{~K}$. We deduce from Eq. (3) the ratio $\rho_{0}^{\downarrow} / \rho_{0}^{\uparrow}$ ranges from 5 at $200 \mathrm{~K}$ to 16 at $4.2 \mathrm{~K}$. The temperature dependence of the ratio $\rho_{0}^{\downarrow} / \rho_{0}^{\uparrow}$ is given by ${ }^{16}$ 


$$
\rho_{0}^{\downarrow} / \rho_{0}^{\uparrow}=\frac{\rho_{r 0}^{\downarrow}+\rho_{0}^{\downarrow}(T)}{\rho_{r 0}^{\uparrow}+\rho_{0}^{\uparrow}(T)},
$$

where $\rho_{r 0}^{\downarrow(\uparrow)}$ is the residual resistivity and $\rho_{0}^{\downarrow(\uparrow)}(T)$ is the $T$-dependence term for spin down (up). Our result is consistent with the fact that in iron the temperature-dependent scattering $\rho_{0}^{\downarrow(\uparrow)}(T)$ is independent of spins ${ }^{16}$ which results in a strong decrease of domain-wall scattering contribution as the temperature is increased. ${ }^{5}$ However, we have not taken into account the dependence of DW thickness with temperature. As seen above, the wall thickness is expected to be enhanced at high temperature. Consequently, this leads also to a decrease of $R_{w}$ with increasing temperature.

*Present address: Institut d'Electronique Fondamentale (URA CNRS 022), Université Paris sud, 91405 Orsay Cedex, France.

†Present address: Centro Español de Metrologia, c/de Alfar, 2, 28760 Tres Cantos (Madrid), Spain.

${ }^{1}$ L. Berger and G. Bergmann, The Hall Effect and its Applications, edited by C. L. Chien and C. R. Westgate (Plenum, New York, 1980).

${ }^{2}$ See, for example, Shufeng Zhang, Phys. Rev. B 51, 3632 (1995).

${ }^{3}$ G. C. Cabrera and L. M. Falicov, Phys. Status Solidi B 61, 539 (1974); 62, 217 (1974).

${ }^{4}$ L. Berger, J. Appl. Phys. 49, 2156 (1978).

${ }^{5}$ P. M. Levy and Shufeng Zhang, Phys. Rev. Lett. 79, 5110 (1997).

${ }^{6}$ J. F. Gregg, W. Allen, K. Ounadjela, M. Viret, M. Hehn, S. M. Thomson, and J. M. D. Coey, Phys. Rev. Lett. 77, 1580 (1996).

${ }^{7}$ U. Ruediger et al., Phys. Rev. Lett. 80, 5639 (1998).

${ }^{8}$ G. Tatara and H. Fukuyama, Phys. Rev. Lett. 78, 3773 (1997).
To summarize a direct study of domain-wall scattering is reported. We demonstrate that this phenomenon allows the possibility of identifying not only the magnetization reversal phenomena but also electronic transport characteristics. In the near future MR measurements on other metallic alloys with strong uniaxial anisotropy will be useful. In particular, in such materials the behavior of the DW thickness as a function of temperature is need.

We acknowledge useful discussions with Peter M. Levy, Andrew D. Kent, and Michel Viret. The authors thank J. P. Renard for helpful comments on this manuscript. This work was support by the European community within the framework of the Training and Mobility of Researchers by Grant No ERBFMBICT972175.

${ }^{9}$ P. Caro, A. Cebollada, F. Briones, and M. F. Toney, J. Cryst. Growth 187, 426 (1998).

${ }^{10}$ M. Hehn, S. Padovani, K. Ounadjela, and J. P. Bucher, Phys. Rev. B 54, 3428 (1996).

${ }^{11}$ V. Gehanno, Y. Samson, A. Marty, B. Gilles, and A. Chamberod, J. Magn. Magn. Mater. 148, 163 (1995).

${ }^{12}$ M. Viret, D. Vignoles, D. Cole, J. M. Coey, W. Allen, D. S. Daniel, and J. F. Gregg, Phys. Rev. B 53, 8464 (1996).

${ }^{13}$ C. Kooy and U. Enz, Philips Res. Rep. 15, 7 (1960).

${ }^{14}$ J. A. Cape and G. W. Lehman, J. Appl. Phys. 42, 5732 (1971).

${ }^{15}$ A. A. Thiele, Bell Syst. Tech. J. 50, 725 (1971).

${ }^{16}$ A. Fert and I. A. Campbell, in Ferromagnetic Materials, edited by E. P. Wohlfart (North-Holland, Amsterdam, 1982), Vol. 3, p. 747.

${ }^{17}$ G. H. O. Daalderop, P. J. Kelly, and M. F. H. Schuurmans, Phys. Rev. B 44, 12054 (1991).

${ }^{18}$ Akimasa Sakuma, J. Phys. Soc. Jpn. 63, 3053 (1994). 